\title{
How to Integrate Security Compliance Requirements with Agile Software Engineering at Scale? Authors' Copy
}

\author{
Fabiola Moyón ${ }^{10000-0003-0535-1371]}$, Daniel Méndez Fernández ${ }^{20000-0003-0619-6027], ~}$ \\ Kristian Beckers ${ }^{3}$, and Sebastian Klepper ${ }^{4}$ \\ 1 Technical University of Munich and Siemens, Germany fabiola.moyon@tum.com \\ 2 Blekinge Institute of Technology, Sweden, and fortiss GmbH, Germany daniel.mendez@bth.se \\ 3 Social Engineering Academy, Germany kristian.beckers@social-engineering.academy \\ 4 Technical University of Munich, Germany sebastian.klepper@tum.de
}

\begin{abstract}
Integrating security into agile software development is an open issue for research and practice. Especially in strongly regulated industries, complexity increases not only when scaling agile practices but also when aiming for compliance with security standards. To achieve security compliance in a large-scale agile context, we developed $\mathrm{S}^{2} \mathrm{C}-\mathrm{SAFe}$ : An extension of the Scaled Agile Framework that is compliant to the security standard IEC 62443-4-1 for secure product development.

In this paper, we present the framework and its evaluation by agile and security experts within Siemens' large-scale project ecosystem. We discuss benefits and limitations as well as challenges from a practitioners' perspective. Our results indicate that $\mathrm{S}^{2} \mathrm{C}$-SAFe contributes to successfully integrating security compliance with lean and agile development in regulated environments. We also hope to raise awareness for the importance and challenges of integrating security in the scope of Continuous Software Engineering.
\end{abstract}

Keywords: Secure Software Engineering. Scaled Agile Framework- Security standards

\section{Introduction}

Security compliance is a major concern for several industries [8]18. Typically, security practitioners (and regulators) hold a holistic view on security affecting people, processes, and technology [20|8|19]. The perspective of practitioners, however, is rather dispersed and security is commonly treated as just another non-functional requirement [17. Security engineering activities are further too often applied in an ad-hoc manner to a limited set of security problems, e.g., vulnerability testing or static code analysis 8 . Security concerns are often mixed with software functionality and limited to specific implementations like authentication or encryption 34.

Integrating security into lean and agile processes further intensifies these issues and constitutes a well-known research problem [35/17/1. This is especially true for large software development projects. One challenge here is to fulfil requirements rigorously to comply with regulations while not limiting the speed and flexibility agile development methodologies promise. However security standards often require a series of processes to define, analyse, and mitigate security vulnerabilities 22] whereas lean and agile methodologies aim at avoiding rigid linear processes. While the agile manifesto states "to value individuals and interactions over processes", "collaboration over contract negotiation", and "responding to change over following a plan" [6], standards explicitly demand documented evidence of responsibilities, agreements, and established development procedures. 
Our research shall provide a perspective for resolving this conflict through Continuous Security Compliance. In particular, we aim at implementing security standard requirements along with agile development methodologies. To this end, we analysed the issue in a large industrial setting and its currently applied norms: the Scaled Agile Framework (SAFe) as well as the IEC 62443-4-1 standard, later we propose a revised framework dubbed $\mathrm{S}^{2} \mathrm{C}-\mathrm{SAFe}$. We chose the IEC 62443-4-1 standard for secure product development, released in 2018 based on previous secure product development standards such as BSIMM [25], ISO27034 21], or Security by Design with CMMI 33. Our framework shall maintain SAFe's perspective on development procedures and principles while capturing the essential requirements of security standards. In this paper, we contribute:

1. The proposal of our $\mathrm{S}^{2} \mathrm{C}-\mathrm{SAFe}$ framework, a security-standard compliant variant of the Scaled Agile Framework.

2. An evaluation of the $\mathrm{S}^{2} \mathrm{C}-\mathrm{SAFe}$ framework in large-scale software development environments. Given that the introduction of SAFe may take up to 8 years in the chosen organisational context, we conduct our evaluation in a preliminary manner focusing particularly on expert interviews.

We conclude our evaluation with the practitioners' perception of the challenges to achieve security compliance in a continuous manner. By sharing these insights, we particularly hope to raise awareness for the importance, but also challenges of integrating security in large-scale software development organisations following lean and agile principles.

\section{Fundamentals and Related Work}

Continuous Software Engineering (CSE) utilises lean and agile principles for a rapid and continuous "flow" of activities across business, development, and operations [16.

In their "Continuous *" model of CSE, Fitzgerald et al. 17] describe Continuous Security and Continuous Compliance as related but separate concerns and activities. Continuous Compliance (CC) seeks to satisfy regulatory compliance standards on a continuous basis rather than a "bigbang" approach to ensure compliance at release time [18/26. Continuous Security (CS) elevates security from non-functional requirement to key concern by efficiently identifying and addressing security issues throughout all processes [16.

Related work discusses the suitability of agile methods for regulated environments 18 or the extensibility of their use [10. With regard to security, authors focus on solving security aspects in agile environments, without considering regulations as focus [9|31|5|4]; or deriving security activities from a regulations perspective but lacking attention to lean and agile environments as well as corporate operating procedures, e.g., product life cycle [1077. Practical concerns of CS are: adapting the development process to security, better eliciting and tracking security requirements, and incorporating assurance into iterations [5].

Separating CS and CC is illustrated by Fitzgerald et al. [18, concluding that agile methods are suitable for security-critical environments, but not yet adopted in regulated environments .

We aim for Continuous Security Compliance (CSC): combining CC and CS through the holistic view of standardisation that spans across people, processes, and technology 20. Regulatory requirements are utilised to derive security activities and therefore integrating security into a process while also making it standards-compliant [28. Further work concentrates on security governance best practices [12]. This is complementary to prior work focused on the technology side, integrating security engineering into agile processes 131811311 , or on the process side, integrating desirable but not standards-compliant security activities [12]32]. 
$\mathrm{S}^{2} \mathrm{C}-\mathrm{SAFe}$ is the result of applying this holistic principle to both a security-critical and a regulated domain: industrial and automation control systems. The result is an in-depth analysis of a security standard (IEC 62443-4-1) followed by the integration with lean and agile development practices represented by the Scaled Agile Framework (SAFe).

IEC 62443 constitutes a series of standards for network and system security published by the International Electrotechnical Commission (IEC). The standard focuses on requirements for component providers for industrial automation and control systems (IACS), part 4-1 describes process requirements for secure product development [23. We reference this part of the standard as "4-1" or "4-1 standard". SAFe is a widely used process framework that scales lean and agile development to large organisations with multiple levels. It furthermore defines the corresponding roles, responsibilities, activities, and artefacts [24.

For such IACS environments our contribution aims to bridge the gap between lean and agile development, practical security, and compliance 34].

\section{$3 \quad \mathrm{~S}^{2} \mathrm{C}-\mathrm{SAFe}$ Framework in a Nutshell}

The overall aim of our work is to improve product development life-cycle by integrating requirements of IEC 62443-4-1 into SAFe, resulting in the "Security Standard Compliant Scaled Agile Framework" ( $\left.\mathrm{S}^{2} \mathrm{C}-\mathrm{SAFe}\right)$. Figure 1 shows how this is achieved by using visual modelling and by merging techniques as presented in our previous work 28. Essential elements of SAFe and 4-1, such as roles, activities, and artefacts, were captured using Business Process Model and Notation (BPMN), a visual modelling language capable of expressing all of these aspects at once. After refining these models separately with expert practitioners, the process framework model is extended with elements from the security standard model, yielding the $\mathrm{S}^{2} \mathrm{C}-\mathrm{SAFe}$ framework. Previously we found that a visual approach allows for more focused reviews than textual representation.

$\mathrm{S}^{2} \mathrm{C}-\mathrm{SAFe}$ describes how requirements of 4-1 can be implemented within SAFe by showing when to involve roles, execute activities, or generate artefacts. It focuses on SAFe's Continuous Delivery Pipeline (CDP), where the actual product development occurs, and makes it compliant with security requirements (SR), secure implementation (SI), and security verification and validation testing (SVV). These scopes address concerns we captured from practitioners such as frequent vulnerability testing, security requirements traceability, or coding standards review. In addition to a CDP model integrated with SR, SI, and SVV, ${ }^{2}$ C-SAFe contains detailed models for each practice. Figure 2 shows an overview of the $\mathrm{S}^{2} \mathrm{C}-\mathrm{SAFe} \mathrm{CDP}$. The full framework is available in the online material associated with this paper 5 .

\subsection{Security Requirements (SR)}

SAFe does not specify where and how to elicit security requirements even though (security) requirements elicitation constitutes a major challenge both in practice and research [14, especially when developing a product threat model and deriving requirements to counter threats [5]15]. $\mathrm{S}^{2} \mathrm{C}-$ $\mathrm{SAFe}$ therefore explicitly considers security requirements at program and team level and makes them part of the Backlog, equal to all other requirements in prioritisation and traceability. Security Experts facilitate analysis but are not primarily responsible. Instead, Product Management,

\footnotetext{
5 https://dx.doi.org/10.6084/m9.figshare.7149179
} 


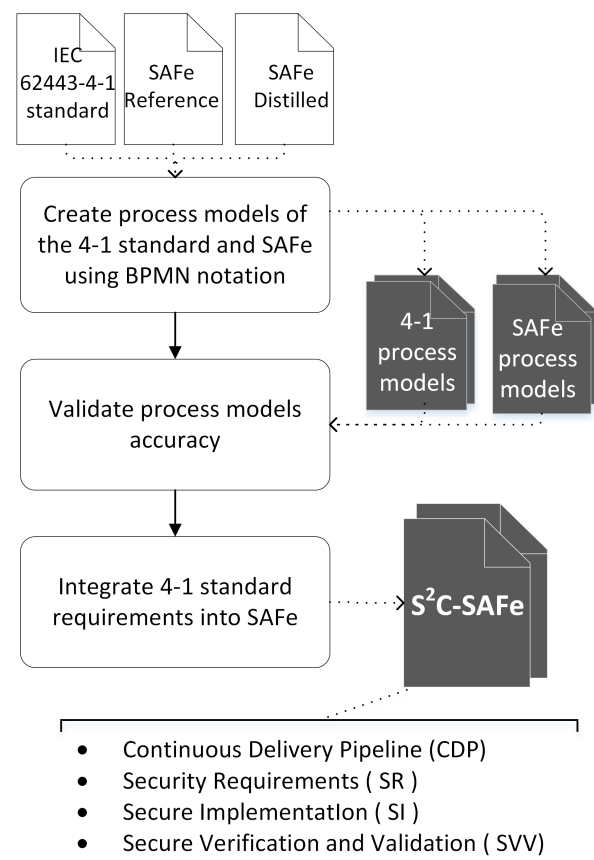

Fig. 1. Creation of $\mathrm{S}^{2} \mathrm{C}-\mathrm{SAFe}$ by generating and merging visual models of $4-1$ and SAFe. Black document symbols designate our contribution. In previous work, we described the integration method [28. The present contribution presents the $\mathrm{S}^{2} \mathrm{C}-\mathrm{SAFe}$ framework and its evaluation.

Business Owners, and Systems Architects are in charge so they become aware of threats. Similarly, the Product Owner requires adequate training to be able to prioritise and approve security requirements.

\subsection{Secure Implementation (SI)}

SI involves following secure coding standards to avoid vulnerabilities. $\mathrm{S}^{2} \mathrm{C}-\mathrm{SAFe}$ follows a process based on coding analysis as introduced in 342 . It defines coding standards early at program level during the PI Planning Event. Security Experts provide guidance so they suit domain and solution. To ensure that coding standards are followed, they are made part of the Definition of Done and agile teams as well as the product owner are trained accordingly.

\subsection{Security Verification and Validation Testing (SVV)}

SVV focuses on detecting and resolving vulnerabilities. One major concern is independence of testers which is enforced through independence rules during formation of agile teams. $\mathrm{S}^{2} \mathrm{C}-\mathrm{SAFe}$ also defines how further activities such as security functionality testing, vulnerability testing, or penetration testing apply to features, user stories, or both. It also defines criteria to keep resource allocation efficient and ensure continuous security testing, placing security functionality testing at team level and conducting all testing activities on program level before every System Demo. S C-SAFe contains 


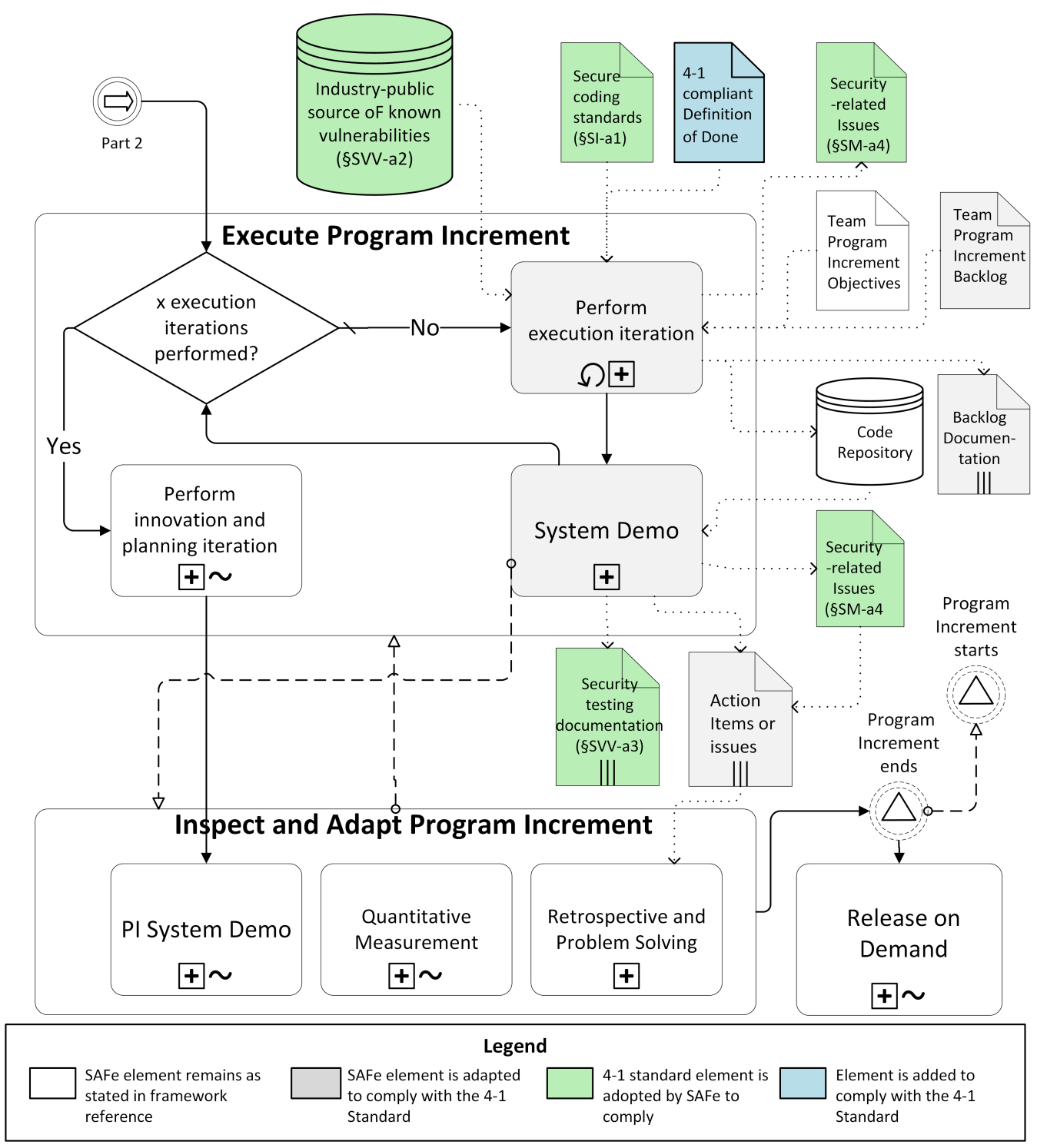

Fig. 2. Excerpt of $\mathrm{S}^{2} \mathrm{C}-\mathrm{SAFe}$ Continuous Delivery Pipeline (CDP). This overview model describes the processes involved to execute and inspect a program increment as described in SAFe plus the artefacts required by the $4-1$ standard in the practices of SR, SI, and SVV.

models that shows a 4-1 compliant SAFe System Demo (see System Demo box in Figure 2). Figure 3 is a more granular refinement showing testing tasks and artefacts, as referred by the 4-1 practice SVV, and their mapping to SAFe roles. Further models are available in the online material. 


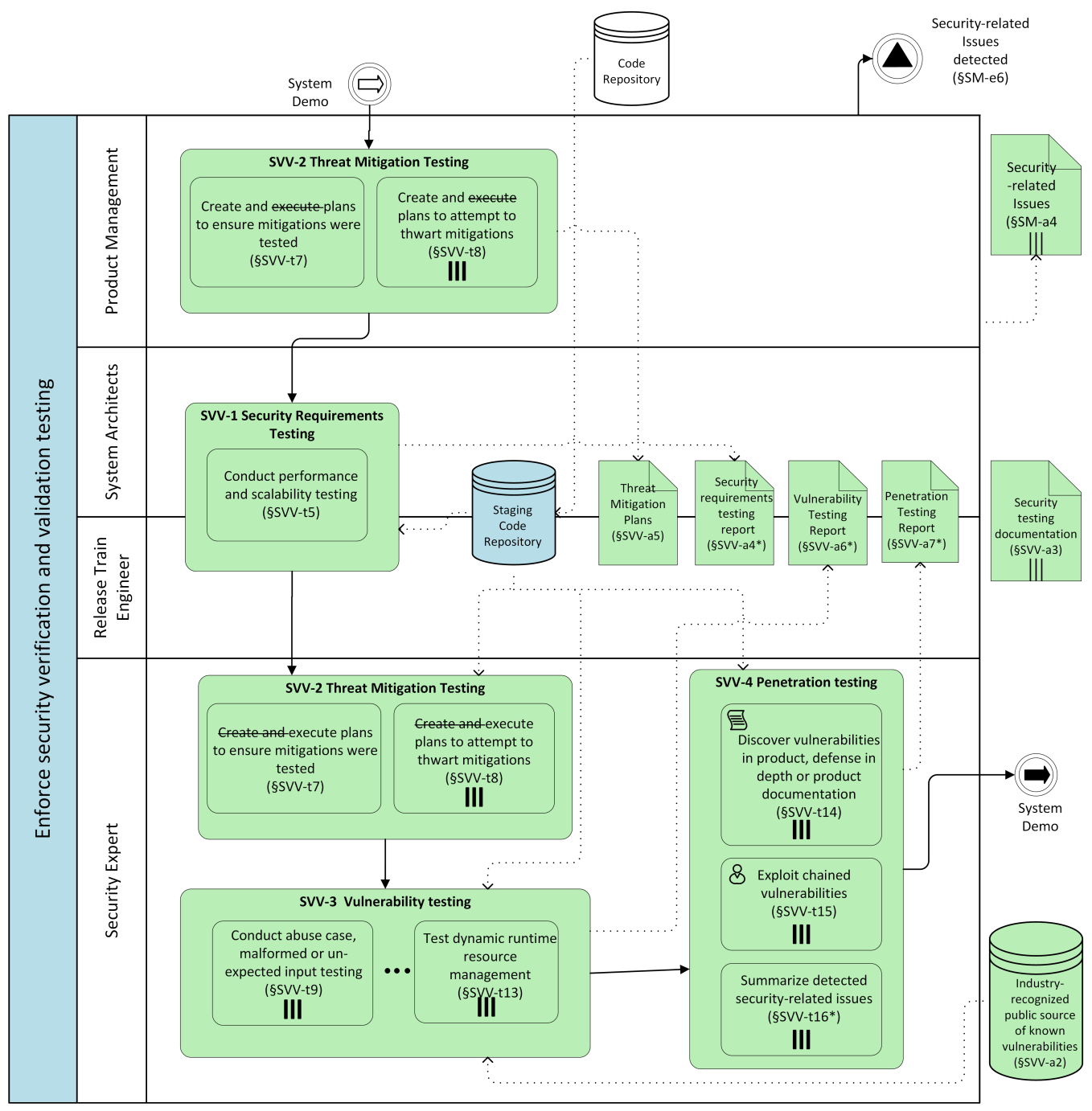

Fig. 3. $\mathrm{S}^{2} \mathrm{C}-\mathrm{SAFe}$ System Demo refinement model. Process diagram that depicts a new activity for SAFe System Demo to perform security verification and validation testing. A Security expert participates for certain types of testing while SAFe Program level actors are also responsible of security testing. Color coding is consistent with Figure 2

\section{Study Design}

We evaluated $\mathrm{S}^{2} \mathrm{C}$-SAFe via expert interviews involving 16 practitioners working at Siemens in security compliance or (agile) software engineering. Among these experts are IEC committee members for 4-1 as well as SAFe core contributors. 
Our overall goal is to explore the meaningfulness of our approach to the needs in a practical context characterised by security-critical and large-scale agile development of software or softwareintensive systems. Our evaluation is guided by the following two research questions:

RQ 1 From the perspective of practitioners, how applicable is $\mathrm{S}^{2} \mathrm{C}-\mathrm{SAFe}$ in this type of environment?

RQ 2 Which challenges do practitioners see when pursuing security compliance in this type of environment?

Our intention is to explore potential benefits and limitations of the here proposed framework. This shall lay the foundation for a roll-out that is minimally disruptive to the organisation and maximally intuitive for practitioners.

\subsection{Subject Selection}

In the industrial environment, where $\mathrm{S}^{2} \mathrm{C}-\mathrm{SAFe}$ is meant to be applied, projects are characterised by large-scale agile practices involving security experts on demand. Since industrial systems are part of critical infrastructure, such projects must comply with formal security standards, like the 4-1 standard when referring to product development. Such projects involve various agile teams with six people each. In those settings projects require direct cooperation between security experts and development teams.

We consciously selected from both groups: development teams working in these settings and security experts joining those projects on-demand, e.g., in conjunction with internal audits.

As these are all experienced professionals, we defined profiles to distinguish their level of expertise according to their key role. Table 1 shows each role's background and share of our 16 interviews. We distinguish top experienced subjects who contribute to the 4-1 standard (Contributor IEC) or to the SAFe framework and its dissemination within the company (Contributor SAFe). We further

distinguish Principle Experts, having vast knowledge and leading teams, Senior Experts, having deep knowledge and guiding colleagues, and finally Experts who are responsible for setting up specific topics into practice.

\subsection{Survey Instrument}

Since our goal is to explore practitioners' opinions about $\mathrm{S}^{2} \mathrm{C}-\mathrm{SAFe}$, we identified semi-structured interviews as the most suitable technique [30. Each interview lasted 1.5 to 2 hours and took place in an isolated environment with one interviewee and two interviewers. One interviewer actively followed the questionnaire and the other one documented the answers and controlled attachment to interview protocol, available at our online material.

Each interview was dedicated to one $\mathrm{S}^{2} \mathrm{C}-\mathrm{SAFe}$ element according to the subject's background: SR, SI, or SVV (c.f. Section 3). Subjects were also introduced to the $\mathrm{S}^{2} \mathrm{C}-\mathrm{SAFe}$ CDP to have an overview of the processes involved the framework as shown in Figure 4.

Interviewers first briefed individual subjects about the interview flow and the purpose of $\mathrm{S}^{2} \mathrm{C}$ SAFe models as well as their hierarchy (overview model and individual practice models) but did not provide any instruction or training on the actual models. Then they showed a textual excerpt from 4-1 and SAFe, followed by the corresponding individual models and finally merged models from $\mathrm{S}^{2} \mathrm{C}-$ SAFe . Subjects rated the perceived usefulness and practical applicability of each representation. Notes from throughout the interview were discussed before the interview's end to complete the picture. 
Table 1. Mapping of interviews to subject profile and background.

\begin{tabular}{lccl}
\hline \multicolumn{1}{c}{ Profile } & $\begin{array}{c}\text { Sample } \\
\text { size }\end{array}$ & $\begin{array}{c}\text { Interview } \\
\text { numbers }\end{array}$ & \multicolumn{1}{c}{ Background } \\
\hline Contributor IEC & 1 & 13 & IACS software life cycle standardisation \\
\hline Contributor SAFe & 1 & 12 & IACS agile development \\
\hline Principal Expert & 3 & $4,5,8$ & $\begin{array}{l}\text { IACS security standards and processes, security life-cycle, } \\
\text { security architecture }\end{array}$ \\
\hline Senior Expert & 4 & $1,2,6,9$ & $\begin{array}{l}\text { Cloud security, methods and tools for secure solutions, cyber } \\
\text { security coaching, security processes improvement, IT security } \\
\text { assessments }\end{array}$ \\
\hline Expert & 7 & $3,7,10,11$, & $\begin{array}{l}\text { IACS agile development, quality compliance, development of } \\
\text { access control systems, data privacy on smart cities, security } \\
\text { design management, DevOps, security tools development, } \\
\text { automated security testing, IT security in critical infrastructure }\end{array}$ \\
\hline
\end{tabular}

\section{$5 \quad$ Study Results}

Evaluation is based on summarising the answers to closed questions and clustering comments and concerns according to commonalities. We further analysed the emphasis of answers to differentiate acceptance vs. conviction, rejection vs. repulsion, and neutrality vs. doubt. Hence, we tabulated answers according to a 9-point Likert scale. In the following, we summarise and interpret our results according to our research questions.

\section{$5.1 \quad$ Subject Knowledge}

In total we selected 16 subjects with different levels of knowledge about 4-1 and SAFe. Figure 5 shows that now all of them know 4-1 but all except one are aware of other security and safety standards such as ISO/IEC 27001 or other standards of the IEC 62443 family. Similarly, not all know SAFe but all are familiar with other agile process frameworks such as Scrum.

\subsection{Applicability of $\mathrm{S}^{2} \mathrm{C}-\mathrm{SAFe}(\mathrm{RQ} 1)$}

Applicability of S2C-SAFe (RQ 1)

We consider two aspects: applicability itself and potential implementation problems. Overall, while all interviewees strongly agree on the potential of using the integrated model as a means to foster discussions with their counterparts, they see potential problems in the integration of security aspects.

\section{Applicability}

$\mathrm{S}^{2} \mathrm{C}-\mathrm{SAFe}$ demonstrates that SAFe can be compliant with the 4-1 standard. All interviewees deem it usable in their environments and expressed their desire to use it for discussion with other practitioners (see Figure 6). They particularly stated that it would provide a common language 


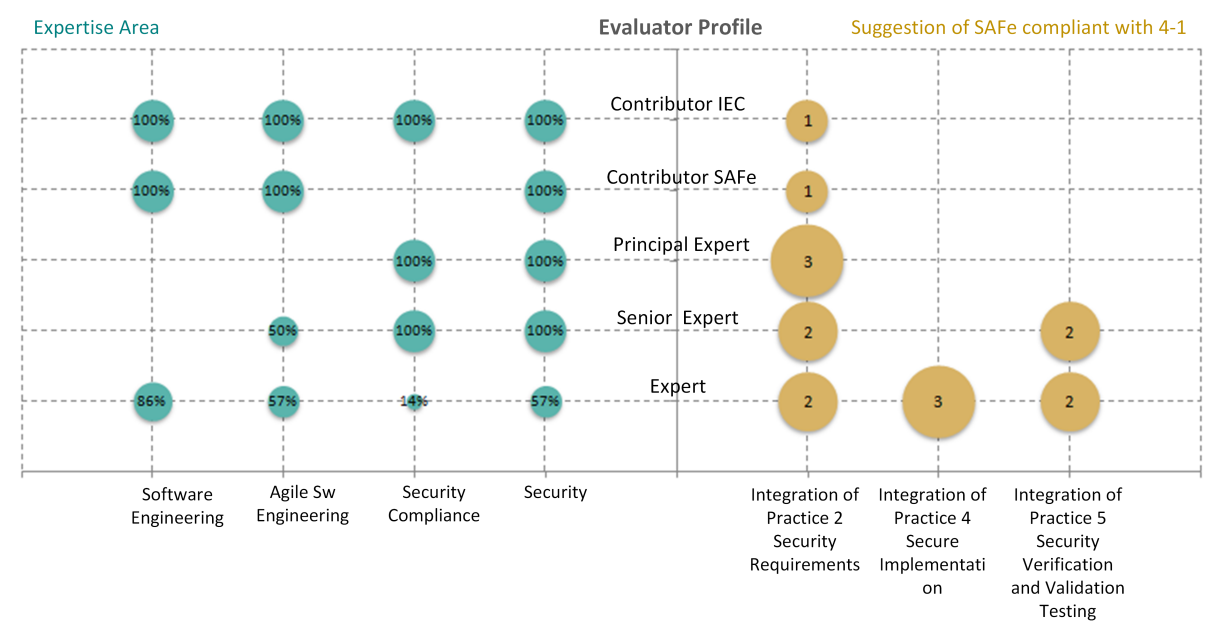

Fig. 4. $\mathrm{S}^{2} \mathrm{C}-\mathrm{SAFe}$ Suggestions distribution into profile groups. Right side: number of interviewees per suggestion. Left side: percentage of interviewees per expertise area.

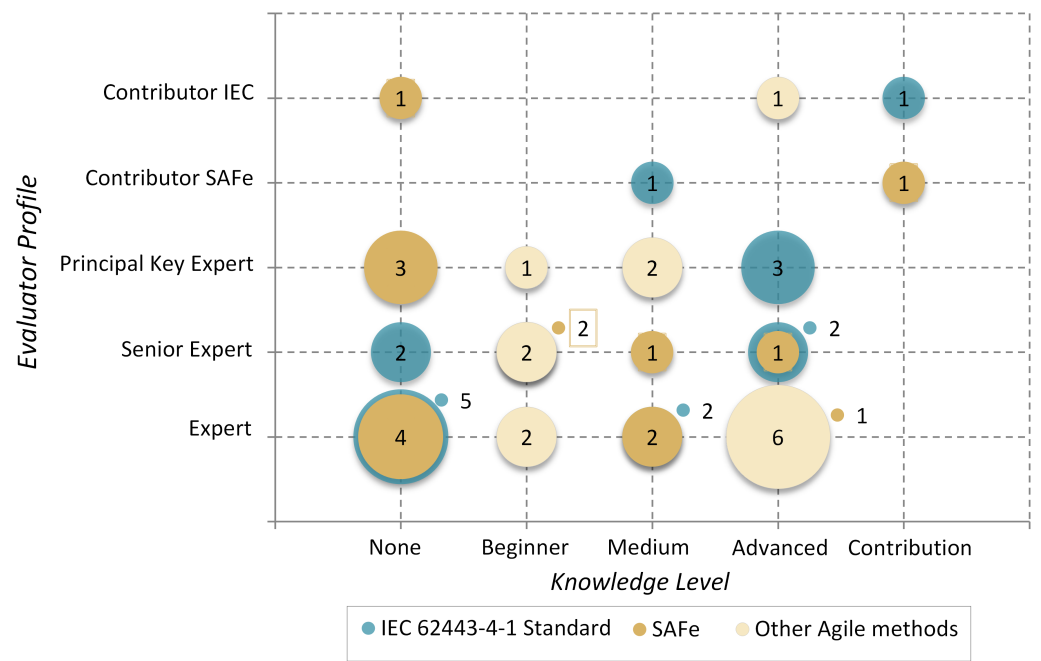

Fig. 5. Subject knowledge of IEC 62443-4-1 and SAFe or comparable process frameworks.

between security and development fields; some even saw it as the only such tool they are aware of (see Table 2). The following paragraphs give detailed results for each of the 4-1 practices introduced in Section 3 .

SR: Subjects strongly agree that this suggestion is feasible. A principal expert (\#8) did not give a positive answer, but instead argued about the complexity of having security experts within teams in general. Almost all envision problems during implementation, most relate to the lack of security practitioners, team security awareness, or split security requirements. Contributor $S A F e$ thinks that proposed security activities overload PI planning while contributor IEC sees no problems if models 


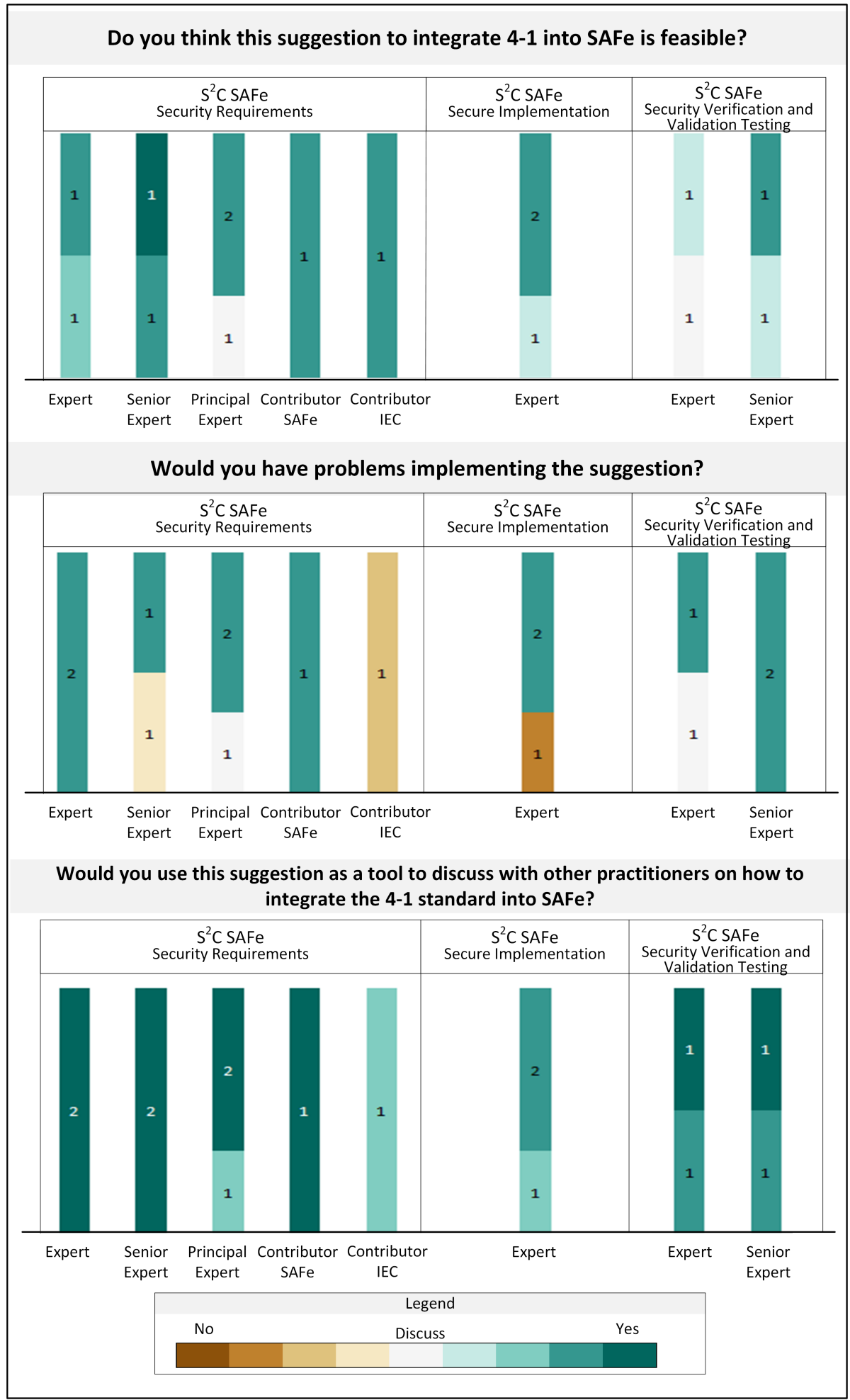

Fig. 6. Summary of opinions about $\mathrm{S}^{2} \mathrm{C}-\mathrm{SAFe}$ applicability based on suggestions regarding 4-1 practices. 
are shown only to people that design processes and not to agile team. However, all subjects plan to use the suggestions as a discussion tool with their respective counterparts.

SI: Subjects strongly agree that this suggestion is feasible. One DevOps expert (\#7) argues that educating the product owner on security is complex. Instead they propose a "security product owner" who would be capable of extending the definition of done (DoD) with security aspects. In contrast, an expert product owner (\#14) remarks the adapted DoD as a key to apply. An expert security consultant (\#11) is confident that problems would exist although they cannot refer to any specific one.

SVV: Although overall positive, opinions on feasibility of this suggestion are not as decided as previously. Two respondents (\#11 expert scrum master and \#1 solutions security senior expert) find the suggestion feasible and well integrated. Another security senior expert (\#6) is concerned about automation support for testing non-functional security aspects and about effort for security practitioners. A security assurance expert (\#3) argues about the role and interactions of security practitioners throughout the process. Hence, all of them envision problems related to the integration of automatic testing, workload, and expertise of security practitioners.

Additionally, as interviewers we experienced that $\mathrm{S}^{2} \mathrm{C}$-SAFe improves communication among practitioners with different profiles and backgrounds. We actively discussed interviewees' issues on security and agile development. All explanations were based on the models we provided. Subjects with the highest level of knowledge (Contributor IEC and Contributor SAFe) challenged us with management or operational questions, e.g., how to implement or even potentially bypass certain aspects. We succeeded in explaining our perspective purely by pointing out specific model aspects. Conversations were dynamic, indicating a common understanding between interviewer and interviewee. Table 2 summarises key opinions on $\mathrm{S}^{2} \mathrm{C}-\mathrm{SAFe}$ while Table 3 lists noteworthy remarks.

Table 2. Summary of key opinions on $\mathrm{S}^{2} \mathrm{C}-\mathrm{SAFe}$.

\begin{tabular}{ll}
\hline Opinion & Interviews \\
\hline Facilitates common language to discuss between security experts and agile team & $2,5,14,16$ \\
\hline Solution is a comprehensible, clear guide & $4,5,7,8$ \\
\hline Increment effort and workload & $5,6,9,12$ \\
\hline Concern about roles expertise to accomplish tasks: Product Owner, Product Management & $3,4,6,10$ \\
\hline Need to increase security awareness & $1,3,7,8$ \\
\hline Concerns on expertise and profile of security experts & $1,4,10$ \\
\hline To have security practitioners within agile teams is challenging & 8,10 \\
\hline Need to have a deep understanding of own process to implement suggestion & 1,16 \\
\hline It is the only tool available & 7,11 \\
\hline Concerns about fit activities into short cycles & 8,12 \\
\hline Find color-coding is useful & 7,13 \\
\hline
\end{tabular}

\section{Potential Implementation Problems}

Our interviewees raised concerns regarding implementation of $\mathrm{S}^{2} \mathrm{C}-\mathrm{SAFe}$ in their project settings. They are particularly interesting to us as they help steering future adaptations and because some 
Table 3. Interviewees' statements on $\mathrm{S}^{2} \mathrm{C}-\mathrm{SAFe}$.

\begin{tabular}{lccc}
\hline Quote & $\begin{array}{l}\text { Inter- } \\
\text { views }\end{array}$ & Profile & Background \\
\hline $\begin{array}{l}\text { Big advantage, we could speak same language as SAFe } \\
\text { experts. This would dramatically reduce problems to adapt }\end{array}$ & 2 & Senior Expert & Security compliance \\
SAFe. Yes, I would love to use it as a discussion tool. & & & \\
\hline $\begin{array}{l}\text { It makes sense what you did. If it is not possible SAFe is } \\
\text { broke }\end{array}$ & 4 & Principal Expert & Security research \\
\hline Sure is feasible, how to measure success I wonder & 5 & Principal Expert & Head security group \\
\hline It is a very nice way to reduce complexity to discuss. & 7 & Expert & DevOps \\
\hline $\begin{array}{l}\text { Visibility of security into agile development environment. } \\
\text { Transparency of what is being achieved }\end{array}$ & 9 & Senior Expert & Security assessments \\
\hline Sure, there is nothing else. I don't think there is anything & 11 & Expert & Security consultant / \\
\hline We need to involve a pilot implementation & 12 & Contributor & $\begin{array}{l}\text { Head development } \\
\text { group }\end{array}$ \\
\hline I will use it as a basis to communication & SAFe & Product Owner \\
\hline I like it. It makes dedicated to think about security & 15 & Expert & Systems Architect \\
\hline
\end{tabular}

concerns are rather general challenges on the integration of security, let alone continuous security engineering. These concerns can be summarised as follows:

\section{Models should guide instead of comprehending compulsory processes}

One senior expert argued that if a model is too strict, people will not adapt it and bypass compliance efforts $(\# 1)$. The suggestions seem difficult to implement in iterations or in specific program increments. This seems particularly true for security testing (vulnerability/penetration) prior to or during a System Demo. This highlights the need for an incremental prototypical implementation of individual suggestions to shed light on potential adaptation barriers which might differ in dependency to the practices and the roles.

\section{Achievement of security expertise and awareness}

During the design phase, we emphasised that $\mathrm{S}^{2} \mathrm{C}-\mathrm{SAFe}$ cannot compensate for a Product Owner with knowledge of 4-1. Our interviewees confirm that this holds not only for general SAFe roles but also for security practitioners in general. Both security and agile development experts agree that security expertise for each part of the solution requires specialisation. Such specificity on profiles would aggravate the deficit of security professionals. Exemplary statements are "During verification of compliance, people tend to deviate from the standard" $(\# 7)$ or "Lack of experience on security compliance leads to failed projects" (\#3, security expert).

Difference between agile and express development delivery

Security is generally perceived to be something that slows down agile development processes. Some exploratory questions revealed that agile time constraints are not followed in our settings, e.g., daily meetings last more that 15 minutes. Our concept of agile therefore seems to relate more to iterative and incremental development than to express delivery and integrating security-related activities will surely expand this gap further. While we understand the need for a trade-off between effort and cost for adapting security (or any other quality facet) this aspect seems particularly hard to achieve and constitutes an open issue. 


\subsection{Continuous Security Compliance Challenges (RQ 2)}

The interviewees were asked to mention priorities among the security activities described in the 4-1 development life cycle. Security requirements (SR) seems to be the most challenging practice for our interviewees. Other priorities differ per profile, as shown in the examples for security management and security verification and validation testing.

The top priority issue is raising awareness for security to achieve continuous security compliance. Second place is taken by an adequate prioritisation of security aspects and common perspectives among management and teams. Challenges for security integration into continuous software engineering seem similar to those with linear development models. Subsequently we summarise our key findings on the challenges raised.

Security requirements elicitation: Challenges go beyond elicitation, from prioritisation over allocating them to increments and tracking adequate testing. Respondents extend the concern to overall 4-1 activities into cycles, e.g., threat analysis, testing, or issue management. Some related quotes include "What does the standard says about iterations and when the required process should occur again?"(\#15, software architect) or "Problem is to identify what is the most important and which things can be done in parallel" (\#12).

Security more than a non-functional requirement: 4-1 contains an overview of security as described by compliance. Our interviewees state that security is normally addressed via functional requirements while other aspects, such as management-related ones, are too often left behind.

Software architecture impact: Software architectures are built incrementally in continuous development. One interviewee argued in particular: "How to have security design or requirements of something we don't know yet, something we create on the go" (\#12, Contributor $S A F e)$. We argue that security analysis can be done while thinking about the goal and later iteratively extend it to the solution-specific components. However, this needs a certain continuity just like other non-functional properties, which project participants seem to see as difficult to achieve.

Improvement demand for security expertise and awareness: In development teams the lack of expertise for security seems to be a common theme 5 . Particularly, our group of interviewees seems to have a sound level or security awareness: "I see the need of security" (\#15, product owner). They comprehend that challenges also depend on the role and therefore some interviewees even suggest to define new (agile) security-related profiles such as a "secure product owner" or a "secure system architect". Furthermore, respondents argue that security expertise should generally be improved to achieve compliance. This is exemplified in the following quote: "A new secure product owner could do it" (\#7). Interestingly, these observations corroborate the need to raise a common awareness for security in the overall agile team: "implementations deviate from standard [and often] lead to fake implementations" (\#2, security compliance senior expert); "There are guidelines to bypass compliance rules" (\#8, security principal expert).

Security compliance as a common agreement: Related to our previous observation is that subjects perceive compliance as a complex endeavour. They noticed that management, teams, and even compliance practitioners have different perspectives on compliance. Some see security compliance as a burnout journey, others as a luxury and others again as a worthwhile goal. A common agreement on the need to achieve common security standards is therefore a prerequisite for the success of our undertaking. 
Misunderstandings of agile engineering terms: In our interviews we noticed that terms are used often in a cumbersome manner. For instance, subjects with agile development knowledge (e.g., $\# 1, \# 2, \# 3)$ often referred to Scrum only implicitly by mentioning specific elements such sprint, iteration, and product owner; "definition of done" was often used when referring to acceptance criteria; other interviewees had difficulties in capturing the notion of artefacts in context of process models: "the word artefact is not easy" (\#10, expert). As a matter of fact, such key concepts are still subject to current debates and need further attention in future work generally dealing with software processes [27.

\section{Conclusion}

In this paper we reported on our work towards integration of security requirements derived from IEC 62443-4-1 into large-scale agile development based on SAFe in order to facilitate CSC. We presented the $\mathrm{S}^{2} \mathrm{C}-\mathrm{SAFe}$ framework and evaluated it based on interviews with 16 industry experts. Evaluation results strengthen confidence that this approach and the resulting models provide a feasibly way for security compliance in large-scale organisations practising lean and agile development.

\subsection{Impact and Implications}

Results show $\mathrm{S}^{2} \mathrm{C}-\mathrm{SAFe}$ models have a clear impact for practitioners. They show precisely how software engineering and security practitioners have to interact to achieve the goal of security compliance. Furthermore, the models can be understood in a time-effective manner and challenge popular belief that agile processes are a gateway to chaos and therefore not reconcilable with security and compliance concerns. The unanimous response to our work was the exact opposite: Introducing large-scale agile processes demands a culture and mindset change. Even though not our intention, the models helped to convey to sceptical practitioners that both secure and agile development is feasible at scale with reasonable effort.

Our research strongly indicates that models are an excellent way to mediate between agile practitioners and security experts. Particularly visual models allowed them to engage the challenge of continuous security compliance together. Moreover, these models pave the way for analysing various further challenges of the research field: Do models increase the speed of adapting large organisations to secure agile processes at scale? Are models a better way of getting security norms accepted in daily software engineering activities? Can models provide guided and precise support for secure agile security governance? We are confident that our contribution supports researchers to further investigate these questions.

\subsection{Relation to Existing Evidence}

Our study is in tune with existing trends of empirical studies on secure software engineering [29, but extends the study population in number and profile. To the best of our knowledge, preceding studies involved up to 11 practitioners with mixed background or students as subjects and focused on valuated, yet isolated topics. An integrated view on a security standard compliant agile framework was not in their scope. Our contribution is aimed at this gap and involves 16 experienced professionals, partially with contributing roles to the standards or decision-making roles in the organisation. We focused on the highest ranking experts available. As explained, a SAFe integration may last up to 8 years and the interviewees are high-ranked professionals. Their opinion is the closest to certainty in a timely evaluation. 


\subsection{Limitations and Threats to Validity}

Qualitative studies inherently carry limitations and interview research in particular has threats to validity that need discussion, the most important of which shall be discussed here.

The individual expertise of each participant might influence their attention and interpretation of security requirements as well as agile practices captured in the models. We tried to mitigate this with discussion-intensive preparation procedures, but also by letting subjects interpret the models as they are without any further instruction. We were interested in potential bias towards the subject

of security compliance as that reflects on the projects where those models shall be applied.

Similarly, involving experts from each respective field carries the risk of self-selection and confirmation bias. To mitigate this we selected subjects according to typical roles in the target organisation environment instead of their particular interest in the topic. The same is true for which part of $\mathrm{S}^{2} \mathrm{C}-\mathrm{SAFe}$ they reviewed (requirements, implementation, or testing). We also designed interview plan and questionnaire accordingly and allocated interviewees to models based on previously defined profiles.

Overall, our study already strengthens our confidence in the capability of $\mathrm{S}^{2} \mathrm{C}-\mathrm{SAFe}$ to integrate security and compliance concerns with lean and agile development. We cordially invite researchers and practitioners to join our endeavour towards facilitating continuous security compliance in large organisations and regulated environments.

\section{Acknowledgements}

To the practitioners that evaluate this work, as well as to Markus Voggenreiter and Florian Angermeir for reviewing.

\section{References}

1. Ahola, J., Frühwirth, C., Kutvonen, L., Helenius, M., Nyberg, T., Pietikäinen, A., Pietikäinen, P., Röning, J., Ruohomaa, S., Särs, C., Siiskonen, T., Vähä-Sipilä, A., Ylimannela, V.: Handbook of the Secure Agile Software Development Life Cycle. University of Oulu, Finland (2014)

2. Baca, D., Boldt, M., Carlsson, B., Jacobsson, A.: A novel security-enhanced agile software development process applied in an industrial setting. In: Proc. of ARES (2015)

3. Baca, D., Carlsson, B.: Agile development with security engineering activities. In: Proc. of ICSSP. pp. 149-158. ACM (2011)

4. Baca, D.: Developing Secure Software -in an Agile Process - Doctoral Dissertation. Blekinge Institute of Technology (05 2012)

5. Bartsch, S.: Practitioners' perspectives on security in agile development. In: ARES (2011)

6. Beck, K., Beedle, M., van Bennekum, A., Cockburn, A., Cunningham, W., Fowler, M., Grenning, J., Highsmith, J., Hunt, A., Jeffries, R., Kern, J., Marick, B., Martin, R., Mellor, S., Schwaber, K., Sutherland, J., Thomas, D.: Manifesto for agile software development (2001)

7. Beckers, K.: Pattern and Security Requirements - Engineering-Based Establishment of Security Standards. Springer (2015)

8. Bell, L., Brunton-Spall, M., Smith, R., Bird, J.: Agile Application Security. Enabling Security in a Continuous Delivery Pipeline. O’Reilly (2017)

9. Beznosov, K., Kruchten, P.: Towards agile security assurance. In: Proc. of NSPW. ACM (2004)

10. Cawley, O., Wang, X., Richardson, I.: Lean/agile software development methodologies in regulated environments - state of the art. LNBP (2010) 
11. Chóliz, J., Vilas, J., Moreira, J.: Independent security testing on agile software development: A case study in a software company. In: Proc. of ARES (2015)

12. Daennart, S., Moyon, F., Beckers, K.: An assessment model for continuous security compliance in large scale agile environments - exploratory paper. In: CAiSE (2019)

13. Felderer, M., Pekaric, I.: Research challenges in empowering agile teams with security knowledge based on public and private information sources. In: Proc. of SecSe (2017)

14. Fernández, D.M., Wagner, S.: Naming the pain in requirements engineering: Design of a global family of surveys and first results from germany. In: Proc. 17th Intl. Conference on Evaluation and Assessment in Software Engineering. ACM (2013)

15. Fernandez, E.B.: Threat modeling in cyber-physical systems. In: Proc. 14th Intl. Conf on Dependable, Autonomic and Secure Computing (2016)

16. Fitzgerald, B., Stol, K.J.: Continuous software engineering: A roadmap and agenda. The Journal of Systems and Software (2015)

17. Fitzgerald, B., Stol, K.J.: Continuous software engineering: A roadmap and agenda. In: The Journal of Systems and Software. vol. 123, pp. 176-189 (01 2017)

18. Fitzgerald, B., Stol, K.J., O'Sullivan, R., O'Brien, D.: Scaling agile methods to regulated environments: An industry case study. In: Proc. of ICSE. IEEE (2013)

19. Humphreys, E.: How to measure the effectiveness of information security (2017), https://ww. iso. org/news/2016/12/Ref2151.html

20. IEC: 62443-1-1 Security for industrial and automation control systems Part 1-1 Models and Concepts. USA, 2014 edn. (2014)

21. ISO/IEC: 27034 Information Technology - Security techniques - Application Security (2011)

22. ISO/IEC: 27001 IT - Security techniques - Information security management systems (2013)

23. ISO/IEC: 62443-4-1 Security for industrial automation and control systems Part 4-1 Product security development life-cycle requirements (2017)

24. Leffingwell, D., Yakyma, A., Knaster, R., Jemilo, D., Oren, I.: SAFe Reference Guide. Pearson, 2017 edn. (2017)

25. McGraw, G., Migues, S., Chess, B.: Building security in maturity model, https://www.bsimm.com/ about.html

26. McHugh, M., McCaffery, F., Fitzgerald, B., Stol, K.J., Casey, V., Coady, G.: Balancing agility and discipline in a medical device software organisation. In: Proc. of SPICE. Springer (2013)

27. Méndez Fernández, D., Böhm, W., Vogelsang, A., Mund, J., Broy, M., Kuhrmann, M., Weyer, T.: Artefacts in Software Engineering: What are they after all? ArXiv e-prints (2018)

28. Moyón, F., Beckers, K., Klepper, S., Lachberger, P., Bruegge, B.: Towards continuous security compliance in agile software development at scale. In: Proc. of RCoSE. ACM (2018)

29. Othmane, L., Jaatun, M., Weippl, E.: Empirical Research for Software Security: Foundations and Experience. CRC (2017)

30. Shull, F., Singer, J., Sjøberg, D.I.: Guide to Advanced Empirical Software Engineering. Springer-Verlag New York, Inc., Secaucus, NJ, USA (2007)

31. Siponen, M., Baskerville, R., Kuivalainen, T.: Integrating security into agile development methods. In: Proc. of HICSS (2005)

32. Stephanow, P., Khajehmoogahi, K.: Towards continuous security certification of software-as-a-service applications using web application testing techniques. In: Proc. of CAINA (2017)

33. Technology, S.A.C.: Security by Design with CMMI for Development Version 1.3. CMMI Institute (2013)

34. Tøndel, I.A., Jaatun, M.G., Cruzes, D.S., Moe, N.B.: Risk centric activities in secure software development in public organisations. IJSSE 8(4), 1-30 (2017)

35. Turpe, S., Poller, A.: Managing security work in scrum: Tensions and challenges. In: Proc. of SecSE (2017) 